Mirai. Estudios Japoneses

ISSN-e: 1988-2378

\title{
[re]Japón. La concepción del espacio turístico: del territorio a la arquitectura
}

\section{Dr. Jin Taira Alonso ${ }^{1}$}

Resumen: Desde el punto de vista del turismo entendido como sector económico, constituye un campo de evidente potencialidad que se encuentra en vías de desarrollo y posicionado en un plan estratégico nacional para multiplicar su tráfico internacional en los próximos años. Esta iniciativa constituye un reto dentro de la tendencia actual de la demanda y la competitividad internacional a la que se enfrenta. Para afrontar este nuevo escenario, Japón debe reestructurar su territorio con el fin de contener la afluencia prevista desde el punto de vista de la movilidad sostenible; la concepción de redes temáticas; la creación, mejora y/o conservación de sus polos de atracción; la innovación de modelos tipológicos de alojamiento turístico que se adecuen a la demanda; y todo ello atendiendo a la necesaria sostenibilidad de un modelo económico que debe estructurarse transversalmente con un enfoque multiescalar de gran flexibilidad.

Japón, consciente especialmente tras el desastre de Fukushima, debe afrontar el futuro atendiendo a procesos sostenibles y resilientes, estudiar modelos arquitectónicos de apoyo al turismo que aporten respuestas responsables al cambio climático a través de modelos bioclimáticos apoyados en el uso de energías renovables, optimizando sus recursos propios en su frágil equilibrio ecológico e incorporando a sus habitantes al compromiso de una hospitalidad más abierta y ambiciosa (omotenashi).

Palabras clave: Diseño Urbano; Planeamiento Urbano; Ecología Urbana; Turismo Sostenible; Capacidad de Carga.

\section{[re]Japan. The conception of tourism space: from territory to architecture}

Abstract: The upward trend of international tourists visiting Japan is currently an ongoing development field of significant potentiality, supported by a national strategic plan in order to multiply its statistics in the coming years. This initiative is a challenge facing the current trend of demand and international competitiveness.

Japan, involved in an economic recession since the early nineties which peaked in 1995 with the economy bubble burst, the Kobe earthquake and the terrorist attacks of the Aum sect, turned again to face a tragic triple scenario that tested the country resistance capacity towards the great Tohoku earthquake, the tsunami and the Fukushima nuclear plant disasters in 11th of March. Facing the urgent need for the material and social reconstruction of the country, Japan has met an unexpected positive economical vector as an international tourism attractor center.

To address this new scenario, Japan must restructure its territory in order to contain the expected influx from the point of view of sustainable mobility; the conception of thematic networks; the creation, improvement and/ or maintenance of attraction nodes; the innovation of typological models of tourist accommodation to suit consumers' demands; and above all, giving response to the necessary sustainability of an economical model that should be structured across a multi-scalar approach of great flexibility, in a call that incorporates its inhabitants in a more open and ambitious, if possible, commitment to hospitality (omotenashi).

Keywords: Urban Planning; Urban Design; Urban Ecology; Sustainable Tourism; Load Capacity.

Sumario. 1. Introducción. 2. [re]Japón. 3. De personas y de necesidades. 3.1. De personas: la evolución del turismo internacional en Japón. 3.2. De necesidades: planificación urbana y diseño identitario en Japón.

$1 \quad$ Universidad de Las Palmas De Gran Canaria.

jin.taira@ulpgc.es 
3.2.1. Territorio. 3.2.2. Movilidad. 3.2.3. Redes. 3.2.4. Polos turísticos. 3.2.5. Arquitectura alojativa. 3.2.6. Infraestructura sostenible. 4. Plan Visión.

Cómo citar: Taira Alonso, J. (2017). [re]Japón. La concepción del espacio turístico: del territorio a la arquitectura, en Mirai. Estudios Japoneses 1(2017), 279-290.

\section{Introducción}

El trabajo de investigación presentado es una extensión de la trilogía de escala convergente de estudios realizados por el autor en el Laboratorio de Investigación de Morfología Urbana del Institute of Industrial Science, The University of Tokyo, dirigidos por el profesor Fujii Akira: [re]DEFINITION ${ }^{2}$ como tesis de máster (1999) en el que se desarrolla a nivel conceptual la naturaleza del cambio; [re]TOKYO ${ }^{3}$ como tesis doctoral (2002), en donde se estudian los procesos de transformación sufridos por la capital de Japón desde su fundación a comienzos del siglo XVII hasta la actualidad y publicada con el apoyo del Support Programme for Publications and Translations on Japan ${ }^{4}$ de la Fundación Japón; y [re]KYOJIMA-2, como trabajo de investigación post-doctoral en el que se aplican los estudios precedentes en el contexto específico del área de Kyōjima 2-chōme localizado en el distrito de Sumida-ku de Tokio ${ }^{5}$ y financiado por la Japan Society for the Promotion of Science (JSPS).

\section{2. [re]JAPÓN}

No es posible desvincular la arquitectura, de la fenomenología urbana y/o de la complejidad paisajístico-territorial en la que se posiciona. Tampoco lo es ignorar su estrecha vinculación a sus dinámicas ecológicas, económicas y sociales. Y todo ello no es menos cierto en el caso de Japón...

El éxito de la apertura consciente de país como territorio de atracción turística internacional viene propiciado en su mayor parte por la coyuntura económica favorable de sus vecinos asiáticos: China, Corea de Sur y Taiwán. Sin embargo, Japón, sumergido en una situación económica en recesión de la que sigue sin recuperarse, ha puesto sus ojos en un mercado que ha presentado unos números de crecimiento tan inesperados como esperanzadores para el Gabinete que lidera el Primer Ministro Abe. Si bien resulta un sector que ha demostrado su capacidad para generar un estímulo importante en economías emergentes, Japón debe de reorientar de manera flexible sus políticas internas para adaptarse a este nuevo escenario.

2 TAIRA, Jin (2001): “[re]Definition”. En: Architecture Institute of Japan: Summaries of technical papers of Annual Meeting Architectural Institute of Japan. F-2, History and theory of architecture. Tokyo, 31 de Julio de 2001. Tokio: Architecture Institute of Japan, pp. 619-620.

3 TAIRA, Jin (2003): “[re]Tokyo”. En: Architecture Institute of Japan: Summaries of technical papers of Annual Meeting Architectural Institute of Japan. F-1, Urban planning, building economics and housing problems. Tokyo, 30 de Julio de 2003. Tokio: Architecture Institute of Japan, pp. 87-88.

4 TAIRA, Jin (2011): [re]Tokio. Gijón: Satori.

5 TAIRA, Jin (2004): “[re]Kyojima-2". En: Architecture Institute of Japan: Summaries of technical papers of Annual Meeting Architectural Institute of Japan. F-1, Urban planning, building economics and housing problems. Nagoya, 31 de Julio de 2004. Tokio: Architecture Institute of Japan, pp. 419-420. 
Toda adopción de un nuevo paradigma conduce a transformaciones, y muchas de ellas profundas. Ya en 1868, el aperturismo japonés en plena emergencia de la Restauración Meiji, condujo a una guerra civil y a una fractura nacional en pos de una modernización que resultó convulsiva en el sistema social y económico de Japón. El país pasó repentinamente de ser un territorio de férreo y prácticamente aislado control feudal, a convertirse en un territorio moderno sumido en una vertiginosa Revolución Industrial.

Sin embargo, la adopción de la cultura y conocimiento occidentales en Japón pasó por su propio sistema de interpretación. De esta manera se consiguió una aceptación autóctona y evitando una distorsión mayor de la que fue víctima la sociedad japonesa en el proceso de modernización del país a finales del siglo XIX.

Nuevamente, Japón se enfrenta a una nueva disyuntiva frente a lo que procede de fuera. No es ya la cultura o el conocimiento, sino el propio individuo foráneo el que invade con divisas su territorio ávido de experiencias efímeras que puede generar situaciones de tensión social por la conversión de su espacio connatural en un territorio temático.

Los beneficios económicos pueden hacer mejorar el balance recesivo del país, sin embargo, el turismo es un sector frágil que exige continua inversión y modernización, adecuándose y adelantándose a las demandas de un mercado altamente competitivo. Lo que obligaría a un escenario de regeneración y reinvención continua. No solo en sus aspectos intangibles, sino en la concreción de un sistema infraestructural formidable que permita contener una alta demanda de turismo internacional en las próximas décadas.

Por su parte, el 3.11 ha provocado en estos años de recuperación un cambio de mentalidad en el país, más consciente de su fragilidad frente a la naturaleza, el valor social de la comunidad y la prioridad pragmática frente a la creatividad superflua.

Todo ello resultará un importante desafío social, económico y medioambiental, en donde la cultura japonesa y su propia idiosincrasia se verán enfrentadas a un nuevo escenario de profunda transformación. Por tanto, es de esperar una metamorfosis de Japón en los próximos años.

\section{De personas y de necesidades}

Cualquier intento serio de hacer funcionar un medio ambiente, debe comenzar con una formulación de las necesidades del usuario. [...] Aceptaremos algo como necesidad si podemos demostrar que la gente en cuestión, una vez dada la oportunidad, trata activamente de satisfacer esa necesidad. Esto implica que toda necesidad, si es válida, es una fuerza activa ${ }^{6}$.

El número de llegadas de turistas internacionales en el mundo fue en 2015 de 1.180 millones de personas (UNWTO, 2015). Comparado con la población de China (1.373.490.000 habitantes), estamos hablando de que una población equivalente al gigante asiático se desplaza por placer a otros países. Y si lo comparamos con la po-

6 ALEXANDER, Christopher (1971): La Estructura del Medio Ambiente. Barcelona: Tusquets Editores. 
blación global, que alcanzó en 2015 la cifra de 7.376.471.981, estamos hablando de que una de cada seis personas en el mundo hace turismo internacional.

Por otra parte, es importante reseñar las expectativas demográficas en Japón en general y las de su metrópolis Tokio en particular. En este sentido, el país registró una población de 126.981 .000 en 2015. Sin embargo, las proyecciones del libro blanco del urbanismo City View Tokyo del Tokyo Metropolitan Government auguran una dramática reducción a 86,7 millones de habitantes en 2060 debido al irrefrenable envejecimiento de la población del país. Mientras el porcentaje de población tokiota mayor de 65 años alcanzaría la alarmante cifra de un $40 \%$ en el mismo año. Nos encontramos en definitiva ante un país menguante o shrinking country.

\subsection{De personas: la evolución del turismo internacional en Japón}

Centrados en las últimas dos décadas, en 1995 Japón recibía 3,8 millones de turistas extranjeros, mientras en 2015 alcanzó la cifra de 19,7 millones, cinco veces más. Destacando la circunstancia del impacto que genera en esta estadística el nuevo mercado emergente asiático, en donde China representa un 26,8\% ${ }^{7}$. Comparativamente, España recibió 68,1 millones de turistas en 2015.

Por otro lado, en tan sólo dos años, 2014-2016, el número de turistas ha venido a duplicar las estadísticas ${ }^{8}$. En enero del 2014 Japón recibió 944.009 turistas frente al 1.851.895 en 2016. Mientras en el mismo bienio, en julio recibió 1.270.048 de turistas frente al los 2.296.500 en el presente año [fig. 1].
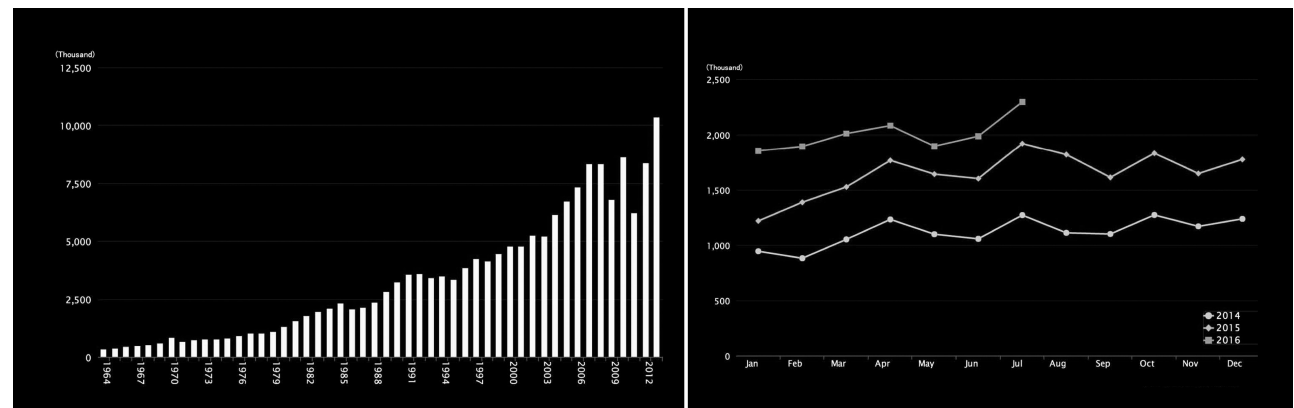

Fig. 1. Evolución en el número de turistas extranjeros en Japón desde 1964 y número de turistas extranjeros en Japón por mes en los últimos tres años. Imagen: Japan Tourism Marketing Co.

El turismo representó en 2015 un 7,9\% del PIB japonés, frente al 11\% aproximado en España, y 29 billones de euros, con un crecimiento del 3,8 \% anual. (Knoema, 2016) ${ }^{9}$

Japan Tourism Marketing Co. (2016): The number of foreign tourists to Japan by month for the past three years. En: Japan Tourism Marketing Co. http://www.tourism.jp/en/tourism-database/stats/inbound [Consulta 04/10/2016].

8 Japan Tourism Marketing Co. (2016): Historical Statistics - Visitor to Japan from Overseas - Tourism Statistics. En: Japan Tourism Marketing Co. http:/www.tourism.jp/en/tourism-database/stats/inbound [Consulta 04/10/2016].

9 Knoema (2015): Japan [página WEB], Knoema. https://knoema.com/atlas/Japan [después de Consulta 20/07/2015]. 
Desde el punto de vista del turismo entendido como sector económico, constituye un campo de evidente potencialidad que se encuentra en vías de desarrollo y posicionado en un plan estratégico nacional para multiplicar su tráfico internacional en los próximos años ${ }^{10}$. Esta iniciativa constituye un reto dentro de la tendencia actual de la demanda y la competitividad internacional a la que se enfrenta el país.

\subsection{De necesidades: planificación urbana y diseño identitario en Japón}

Para afrontar este nuevo escenario, Japón debe reestructurar su territorio transversalmente con un enfoque multiescalar de gran flexibilidad con el fin de contener la afluencia prevista.

El plan visión del gobierno de Abe, busca alcanzar un beneficio de 600 trillones de yenes a través de 10 objetivos para reactivar la economía turística del país. Entre ellos, abrir los palacios de Kioto y Akasaka al público, remodelar los parques nacionales para proveer de una experiencia «interactiva», o mejorar el paisaje de los principales focos turísticos. El plan además busca atraer a más turistas de Europa, EEUU y Australia, así como aquellos de otras nacionalidades de perfil económico alto. Se suavizaría la prohibición sobre alojamientos privados y mejoraría el proceso de inmigración en aeropuertos con la introducción de nuevas tecnologías ${ }^{11}$.

Si bien es cierto que estas medidas podrían incentivar el mercado turístico internacional japonés, la necesidad de activar un plan visión integrado y transversal de actuaciones coordinadas y coherentes resulta indispensable para favorecer un marco de turismo sostenible en Japón. Por ello, y entrando en consideración el nuevo e inesperado escenario, presentamos el plan de actuaciones que deben planificarse, diseñarse y gestionarse desde la perspectiva de la ordenación del territorio y el urbanismo.

En este sentido proponemos la valoración en base a categorías temáticas críticas, que habrán de planificarse para su posterior diseño y adecuación a la naturaleza intangible de la identidad japonesa. Por una parte, las características ambientales y territoriales, y por otra los aspectos relativos a la movilidad, la planificación de redes, los polos de atracción turística, la adecuación de su arquitectura y la gestión sostenible de un sistema territorial sujeto a la amenaza del crecimiento de la demanda turística y su capacidad de carga.

\subsubsection{Territorio}

La primera línea de respuesta a un programa de necesidades es la contextualización del objeto de estudio y por tanto de la concreción de la propia naturaleza de su territorio, que en el caso de Japón se ha visto modificado en múltiples procesos de transformación: seccionándolo, ampliándolo, reduciéndolo, reconfigurándolo, redelimitándolo... en definitiva, construyendo una nueva definición de su extensión territorial, como son las operaciones de ganar terreno al mar en la bahía de Tokio.

10 Ministry of Land, Infrastructure, Transport and Tourism (2014): White Paper on Land, Infrastructure, Transport and Tourism in Japan, 2014. Tokio: Ministry of Land, Infrastructure, Transport and Tourism.

11 Japan Times (2016): Japan doubles overseas tourist target for 2020 [página WEB], Japan Times, 2017. http:// www.japantimes.co.jp/news/2016/03/30/national/japan-doubles-overseas-tourist-target-2020/ [después de Consulta 30/03/2016] 
En este sentido, podemos afirmar que Japón, como objeto de estudio, es un territorio de límites indefinidos en continuo proceso de cambio. Su litoral ha sido prácticamente transformado en su totalidad. En particular, la bahía de Tokio en donde se concentran cuarenta millones de habitantes, es decir, un tercio de la población de Japón, constituye un territorio transformado que ha ido progresivamente ganando terreno al mar desde su adopción como capital administrativa por el clan Tokugawa en 1603. Si bien ha sido objeto de numerosos proyectos de ingeniería, fue sin duda la propuesta metabolista del arquitecto japonés Tange Kenzo con su Plan de Tokio 1960 el más radical y sin embargo el más detalladamente estudiado de las propuestas realizadas hasta ese periodo en la historia de la planificación y el diseño urbano japonés. Su plan se organizaba en un eje longitudinal de autopistas en estructura de anillo que rodeando y partiendo del palacio imperial, en el centro de la metrópolis, cruzaba la bahía hasta alcanzar la costa opuesta en el área de Chiba, una propuesta que nunca llegó abandonarse del todo. No fue por tanto extraño el plan redactado nuevamente por el propio Tange en 1986. Un proyecto desde predicamentos más conservadores, pero con iguales intencionalidades, conectaba Tokio y Chiba a través de un similar eje de movilidad, en este caso apoyado en nuevas islas artificiales. Este proyecto se ejecuta en la actualidad. La última vuelta de tuerca de esta visión es la propuesta de la compañía KPF con su proyecto Next Tokyo del 2016 de una nueva ordenación de islas artificiales posicionadas más al sur del proyecto original de Tange, en donde se plantea un sistema de control resiliente, que incluye una desproporcionada torre de oficinas, y huertos marinos [fig. 2].
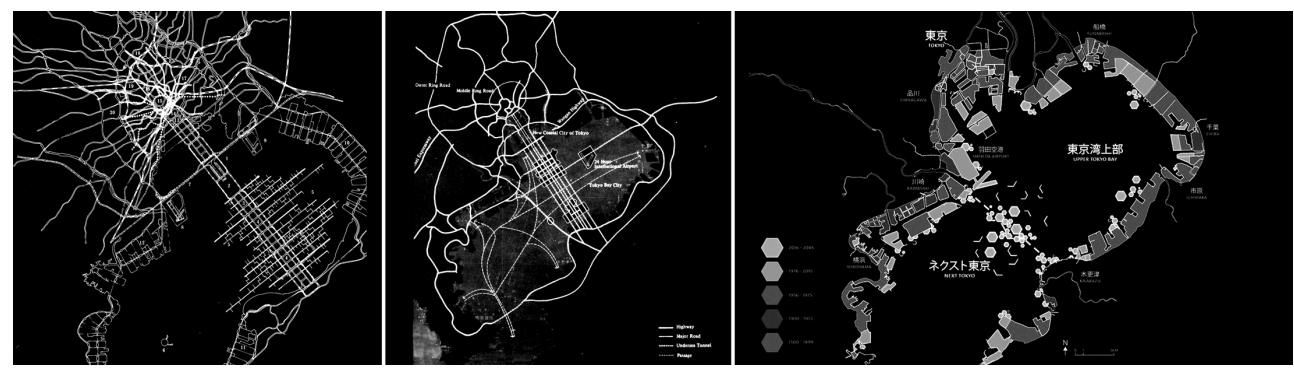

Fig. 2. Planes para la Bahía de Tokio, de izquierda a derecha: Kenzo Tange: Tokyo Plan, 1960 de. Imagen Kenzo Tange \& Associates; Kenzo Tange: Tokyo Plan, 1986. Imagen Kenzo Tange \& Associates; KPF: Next Tokyo, 2016. (C) KPF.

\subsubsection{Movilidad}

En relación a la movilidad, se deberá planificar la accesibilidad, conectividad y capacidad de carga de sus redes. Ello constituirá la necesidad de proyectar sistemas generales de apoyo para asumir este nuevo reto, incluyendo la creación y/o ampliación de terminales, aeropuertos e intercambiadores. La construcción, extensión y mejora del viario existente, así como la inversión en innovación tecnológica aplicada a sistemas de transporte público.

Para un país cuyo acceso se produce por aire o mar, es fundamental el desarrollo y mejora de su infraestructuras aeroportuarias y portuarias. Especialmente teniendo en 
cuenta el horizonte de incremento de turistas extranjeros y el impacto puntual de las olimpiadas de Tokyo 2020. En este sentido, Narita ha comenzado a modernizar sus instalaciones interiores como es el caso de la nueva Terminal-3 diseñada por Nikken Sekkei, Ryohin Keikaku y Party. Haneda pasa a convertirse en un aeropuerto internacional. Mientras la base militar aérea estadounidense de Yokota pretende incorporarse a esta estrategia aeroportuaria metropolitana proponiendo un uso mixto de sus instalaciones. Otras prefecturas de Japón desarrollan con éxito distintas estrategias en sus aeropuertos permitiendo nuevas alternativas de acceso a Japón por otras vías desde sus países vecinos, posibilitando diversificar la excesiva centralización de la movilidad de Tokio, teniendo en cuenta la problemática añadida de ser uno de las zonas del mundo con mayor densidad urbana.

Las cinco rutas Gokaidō que partían de Edo, se han ramificado en la actualidad y desarrollado para configurar un complejo sistema de transporte nacional. En el caso específico de Tokio, si bien la primera transformación de su viario se produce cuando la ciudad gana "sus ruedas"12, haciendo perder parcialmente su carácter veneciano ${ }^{13}$, el momento más drástico se produce con motivo de la celebración de las Olimpiadas de 1964, cuando la capital se convierte en el centro de una red de autopistas que irradian desde Nihonbashi, o kilómetro cero de Japón, ocupando parcialmente los canales y dando lugar al nacimiento de una ciudad tridimensional junto a la emergencia de los problemas medioambientales generados en los canales.

En Tokio, continúa el desarrollo estratégico del viario metropolitano basado en un sistema concéntrico de tres anillos, interconectados entre sí: el interno Tokyo Metropolitan Expressway Central Circular Route, el central Tokyo Outer Loop Road, y el anillo exterior de la National Capital Regional Central Loop Road ${ }^{14}$.

Por otra parte, y con el objetivo de los Olimpiadas de 2020 en el horizonte, Tokio plantea la incorporación del sistema viario de Bus Rapid Transit (BRT), en una versión reducida del sistema de movilidad sostenible promovido por $\mathrm{Cu}$ ritiba (Brasil) con el fin de mejorar la conexión entre las nuevas islas artificiales de Odaiba y el centro de la capital. Por otra parte, ha surgido recientemente la propuesta 400 kilómetros lineales de carril bici que se pretenden crear o implementar en la ciudad ${ }^{15}$.

Japón ha desarrollado mejoras en la red de transporte ferroviario de larga distancia como la conexión Tokio-Kanazawa (Hokuriku Shinkansen Line) y así como el desarrollo tecnológico de nuevos modelos de transporte como el tren Maglev (magnetic levitation) de alta velocidad.

En definitiva, Japón deberá materializar nuevas vías, y rutas, así como crear o implementar los sistemas de acceso insulares. Deberá seguir estrategias formales como la renovación, la ampliación, o la incorporación de innovación tecnológica; atender a las amenazas de impacto territorial sobre el medio ambiente mientras da servicio a la emergencia creciente del flujo de turismo internacional [fig. 3].

\footnotetext{
Waley, Paul (1991): Tokyo: City of Stories. Nueva York: Weatherhill Inc.

Jinnai, Hidenobu (1987): Ethnic Tokyo. Process. Architecture, Vol. 72. Tokio: Process Architecture Pub. Co.

Tokyo Metropolitan Government (2014): Creating the Future: The Long-Term Vision for Tokyo. Tokio: Tokyo Metropolitan Government.

15 Tokyo Plans 400km of Safe Cycling Routes. Because The Olympics [página WEB], Tokyo by Bike. http://www. tokyobybike.com/2015/05/tokyo-plans-400km-of-safe-cycling.html [después de Consulta 20/07/2015].
} 


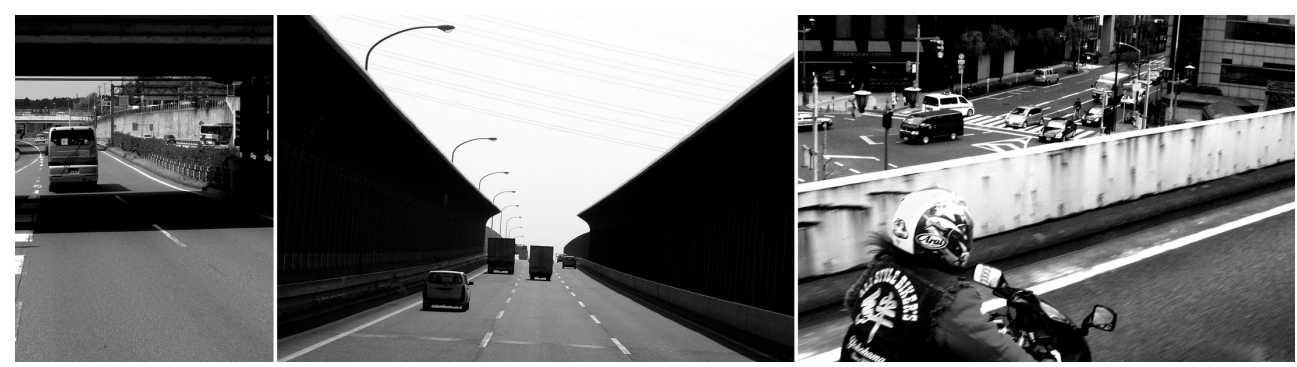

Fig. 3. Paisaje de Movilidad Urbana. (C) Jin Taira.

\subsubsection{Redes}

El espacio urbano arquitectónico incluye dos clases de existentes, el espacio vacío y los objetos sólidos.

C. Rowe

Las políticas para asentar redes temáticas serán fundamentales para consolidar un producto turístico innovador, diferenciado y diverso. La puesta en valor de sus potencialidades tangibles/intangibles afectará directa/indirectamente a la concreción de modelos de apoyo para la consolidación de estas redes. Con ello nos referimos tanto a la concreción de tejidos temáticos como a sus respectivos polos o elementos de atracción, como por ejemplo los centros de interpretación, o conjunto de servicios anexos.

La red urbana territorial es categorizada por su sistema de movilidad. Los tejidos urbanos surgen principalmente de su propósito territorial dando lugar a estructuras interconectadas. De esta manera, los contextos urbanos japoneses surgen como ciudades imperiales, dispuestas en un sistema cartesiano según los preceptos de la geomancia china como Nara y Kioto; ciudades-castillo como Osaka o Tokio, ciudades templo; ciudades portuarias; ciudades-mercado o ciudades-parada.

Frente al tejido materializado, existe el tematizado, es decir el tejido de usos o programado. Por ejemplo, con el fin de diversificar el mercado turístico y las potencialidades de cada región de Japón, diferentes asociaciones bajo la supervisión del ministerio, han desarrollado temáticamente diferentes redes para diversificar el modelo de la Golden Route (conexión Tokio-Kioto). Estamos hablando de nuevas fórmulas turísticas con su propio tejido temático territorial, que incorporan su propia imagen de marca. Es el caso de Hokkaidō con Explore The Wonderland of Eastern Hokkaidō; Tōhoku con la iniciativa Exploration to the deep north of Japan; Kanto con Tokyo \& Around; Chubu, con su campaña de 6 rutas Shoryudō; Kansai con su propuesta Hanayaka Kansai-The Flower of Japan; Chūgoku con The Inland Sea; Shikoku con su Spiritual Island - Shikoku Henro; y Kyūshū con su propuesta Onsen Island.

Por su parte, Tokio reestructura la ciudad temáticamente de acuerdo a su nueva estrategia de planeamiento para su año olímpico siguiendo la lógica de un doble anillo que engloba dos áreas diferenciadas y complementarias: la histórica (Historical Zone) y la nueva (Tokyo Bay Zone) localizada en la zona de la bahía. Mientras, 
se incorporan extensiones metropolitanas hacia el oeste de la capital, o territoriales hacia el norte y sur de las áreas metropolitanas.

Al mismo tiempo Tokio sigue potenciando su papel estructural financiero en sus $h u b s$ en el área de sus veintitrés barrios especiales, mientras emerge con fuerza un nuevo tejido de innovación en el área de Tama.

En definitiva, Japón optimiza su sistema de redes territoriales otorgándoles un contenido intangible turístico que permita diversificar y descentralizar las rutas existentes [fig. 4].
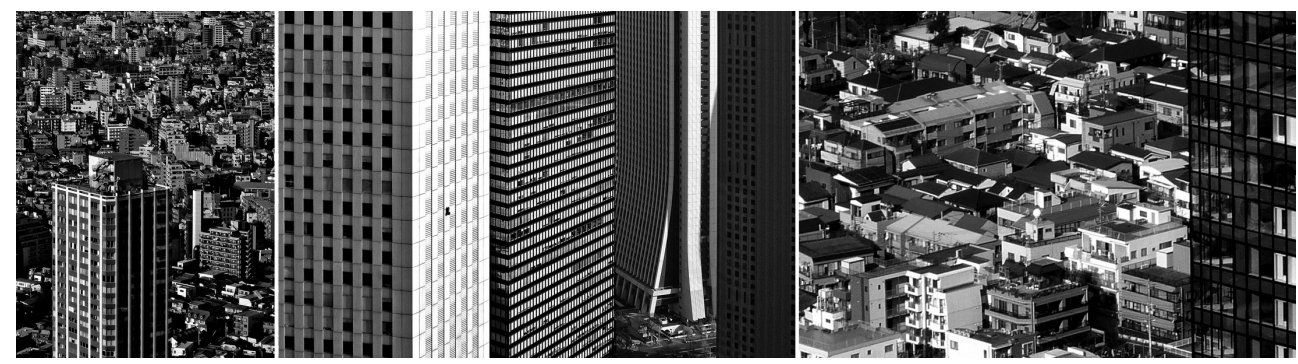

Fig. 4. Paisaje de Estructura Urbana. (C) Jin Taira.

\subsubsection{Polos turísticos}

Por su parte, Japón dispone de un gran patrimonio natural de gran atractivo paisajístico acentuado por polos de atracción turística, como la singularidad de sus templos, o los centros de aguas termales. La atracción generada por el turismo de ciudad como Tokio o Kioto merecen mención aparte, pues constituyen polos de arquitectura tanto clásica como contemporánea, sujeta esta última a una iniciativa por parte de los estudios de arquitectura de gran innovación conceptual y tecnológica admirada y seguida a través de las publicaciones especializadas.

Surgen en este contexto los nuevos proyectos para las Olimpiadas de Tokio, así como los de mejora de distritos de la capital como el Plan Renovación de Aéreas Urbanas definidas por el TMG como Special Urban Reinasance District, y Urban Reinassance District. Entre ellos destacan los trabajos de desarrollo y gestión del distrito OMY (Ōtemachi, Marunouchi, Yūrakuchō), en donde se han llevado a cabo estrategias de redistribución de la edificabilidad para proteger el patrimonio de la estación de Tokio, (diseñada por el arquitecto Tatsuno Kingo en 1914); y sostenibles como la conservación del arbolado, la prevención de la polución del aire, la asistencia para la reconstrucción, la mitigación del calentamiento global, las consideraciones medioambientales y la promoción del reciclaje.

Otras propuestas arquitectónicas han sido menos afortunadas a la hora de poder transferir su edificabilidad a parcelas colindantes. Tal es el caso de la propuesta de Kuma Kengo para el Kabuki-za, en donde el histórico teatro ha debido asumir una torre de oficinas en la parte posterior de su parcela de unos 145 metros de altura.

No sólo la arquitectura se convierte en condensador urbano. La propuesta urbana del arquitecto Rafael Viñoly para el Tokyo International Forum de 1996 incorporaba una calle peatonal arbolada entre el espacio del hall y sus cuatro auditorios, que se ha convertido en un espacio público de gran afluencia. Esta emergencia europea 
de «urbanismo amable» encuentra su continuación en propuestas como los POPS (Privately Owned Public Space) en los nuevos desarrollos urbanos de Tokio como Roppongi Hills o Toranomon Hills; la terraza en la cubierta de la Tokio Plaza de Omotesandō diseñada por Nakamura Hiroshi o la propuesta de Kobayashi Takeshi para la Yoyogi Village, en donde apoyado en contenedores y otras estructuras prefabricadas organiza un sofisticado espacio ajardinado al que se incorporan áreas comerciales, de trabajo y restauración.

En definitiva, Japón se enfrenta a una nueva amenaza por la demanda turística de sus espacios naturales a través de la injerencia expansiva antropizada, ya sea rural o urbana. Frente al esquivo sentido del espacio urbano como lugar de encuentro frente a los entornos ligados al consumo, surgen con fuerza otras propuestas bajo la idea de gentle urbanism o urbanismo amable de precursores como Jan Gehl que asesoran al TMG en la actualidad [fig. 5].

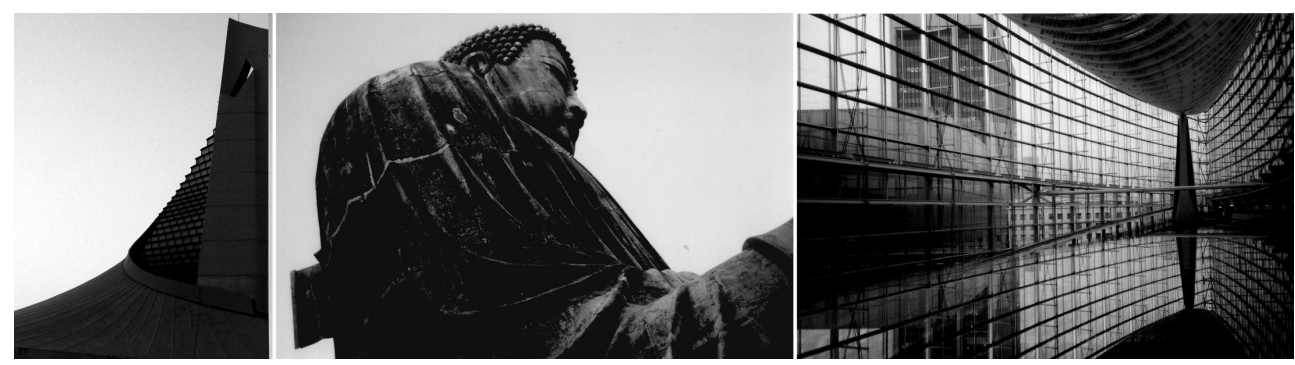

Fig. 5. Paisaje de Polos de Atracción. (C) Jin Taira.

\subsubsection{Arquitectura alojativa}

Frente a la planificación de una demanda de dieciocho millones de turistas en 2016, Japón se enfrenta a la necesidad de incrementar su oferta hotelera, promover nuevos modelos de hostelería, e innovar en posibles sistemas que permitan afrontar la demanda abierta a un rango más amplio.

La arquitectura japonesa se caracteriza por demostrar una gran innovación en propuestas sobre el concepto de habitar. Sin embargo, ese optimismo creativo en el contexto arquitectónico comienza a difuminarse a favor de soluciones más eficientes y menos creativas, especialmente después del 3.11. En este sentido surgen a modo de ejemplo alojativo, nuevas ideas que estimulan un turismo muy específico. Tal es el caso del Hotel Henna atendido por robots; el New Japan Capsule Hotel en Osaka, que ofrece habitaciones cápsula más lujosas; la cadena de hoteles $9 \mathrm{~h}$ Ninehours que aportan una estética de diseño más contemporáneo-cool; o las habitaciones tematicas de Hello Kitty en el Keio Plaza de Tokio.

Otra preocupación emerge con la capacidad de generar una oferta identitaria sin caer en el pastiche. Un ejemplo de ello es la acertada propuesta del nuevo centro turístico de Asakusa diseñada por el arquitecto Kengo Kuma.

En definitiva, Japón debe incrementar y posicionar su oferta alojativa basándose en las expectativas de demanda. Para ello debe redefinir y diversificar sus tipos «endémicos» como el ryokan, o el hotel cápsula mientras innova en tecnología o per- 
sonalización de su producto de hostelería, sin abandonar un lenguaje arquitectónico identitario.

\subsubsection{Infraestructura sostenible}

Finalmente, Japón es consciente, especialmente tras el desastre de Fukushima, de la necesidad de afrontar el futuro atendiendo a procesos sostenibles y resilientes, estudiar modelos arquitectónicos de apoyo al turismo que aporten respuestas responsables al cambio climático a través de modelos bioclimáticos apoyados en el uso de energías renovables, optimizando sus recursos propios en su frágil equilibrio ecológico e incorporando a sus habitantes en el compromiso de una hospitalidad más abierta y ambiciosa.

Tras la tragedia del 11 de marzo, muchos de los sistemas de contención de tsunamis fueron insuficientes para proteger los asentamientos más expuestos, lo que ha generado un debate entre la ordenación de un sistema urbano-paisajístico más resistente (en forma de muros) o resiliente (permitiendo el paso del tsunami). Sin embargo, el apego a la propiedad privada impide soluciones que garanticen la seguridad de la población en el caso de las segundas, y parece imponerse la alternativa de contención tecnológica.

En el caso de Tokio, parte de su territorio como el distrito de Sumida se encuentra por debajo del nivel freático, manteniéndose gracias a un sistema de canales y esclusas que se optimiza con nuevas infraestructuras como el Metropolitan Area Outer Underground Discharge Channel la mayor del mundo en su finalidad.

Todas las ciudades japonesas disponen de un plan de seguridad en caso de terremoto e incendios, garantizando soluciones de emergencia en todas sus escalas, incluyendo los protocolos de evacuación de edificios, localización de rutas de escape y localización de áreas de seguridad por barrios.

Por otra parte, y desde la perspectiva paisajística de los sistemas de infraestructuras, se debe reseñar la necesaria replanificación de su impacto negativo en el visitante foráneo. Por ejemplo, el tendido eléctrico en entornos turísticos de gran afluencia, son algunos de estos aspectos.

En definitiva, Japón continúa optimizando su territorio en una estrategia de seguridad y protección con sus sistemas de infraestructuras urbanas en una incansable estrategia resiliente. Desde la perspectiva del turista es preciso replantear la eficiencia de estas soluciones de ingeniería urbana por propuestas que permitan mejorar las condiciones de impacto negativo de su paisaje, sin olvidar las implicaciones económicas, ambientales y socio-culturales de esas alternativas [fig. 6].

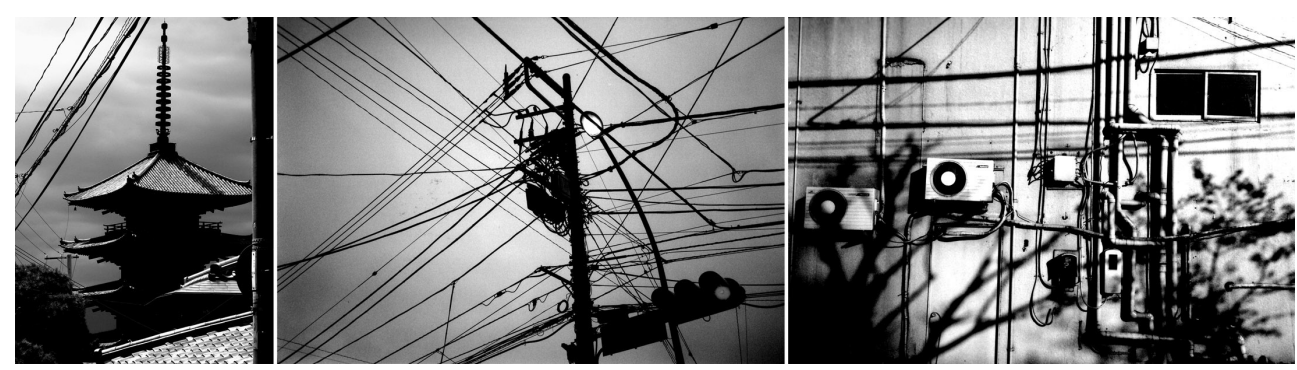

Fig. 6. Paisaje de Infraestructuras Urbanas. (C) Jin Taira. 


\section{Plan visión}

Tokio posee un plan visión ${ }^{16}$, que está coligado a una componente esencial de seguridad, esto es, a su capacidad de respuesta frente a situaciones de emergencia, rehabilitación, recuperación y reconstrucción de un país expuesto a la necesaria organización de protocolos frente a la amenaza.

El plan estratégico del turismo que debe asumir Japón tiene que permitirle encuadrar proyectos en un sistema direccionado flexible y abierto, un plan que habrá de adecuarse al nuevo escenario como atractor turístico internacional.

En definitiva, Japón habrá de diseñar un plan transversal en lo económico, integrador en lo legal, que permita incorporar un sistema comparativo como modelo, construyendo un sistema legible en su información, integral en el grado de su intervención, multiescalar en su concepción, permitiendo una visión que sea identitaria, y flexible para proponer un producto diversificado. 